\title{
ON QUASI-REFLEXIVE BANACH SPACES
}

\section{Y. CUTTLE}

1. Introduction. In the first part of this paper we study some properties of Schauder bases in quasi-reflexive Banach spaces. It is shown that if a quasi-reflexive space of order 1 has a Schauder basis, this basis must be either shrinking or boundedly complete. This result should be compared with the well-known theorem which states that if a reflexive Banach space has a Schauder basis, then this basis must be both shrinking and boundedly complete. If a quasi-reflexive space has a shrinking (boundedly complete) Schauder basis, then its $n$th conjugate space has a boundedly complete (shrinking) basis if $n$ is odd or a shrinking (boundedly complete) basis if $n$ is even. Furthermore if the first conjugate space of a quasi-reflexive space has a boundedly complete basis, then the space itself has a shrinking basis.

The last two sections give conditions under which a quasi-reflexive space is in fact reflexive: if every continuous linear functional attains its supremum on the unit sphere, or if the space is either smooth or rotund, then it is reflexive.

2. Definitions and notation. Let $B$ be a Banach space, $B^{*}$ and $B^{* *}$ its first and second conjugate spaces. The canonical isomorphism of $B$ into $B^{* *}$ will be denoted by $\pi$. If $A$ is a subspace of $B$ we will denote by $A^{+}$the annihilator of $A$ in $B^{*}$. Following [1] we define $B$ to be quasi-reflexive of order $n$ if $B^{* *} / \pi B$ is (finite) $n$-dimensional.

Let $w$ denote the set of positive integers. A sequence $\left(x_{i} ; i \in w\right) \subset B$ will be called a basis if for each $x \in B$ there exists a unique seqence of real numbers $\left(a_{i} ; i \in w\right)$ such that $\lim _{n \in w}\left\|\sum_{i \leq n} a_{i} x_{i}-x\right\|=0$; we then write $x=\sum_{i \in w} a_{i} x_{i}$. A basis $\left(x_{i} ; i \in w\right)$ for $B$ will be called boundedly complete if for each sequence $\left(a_{i} ; i \in w\right)$ of real numbers such that the sequence $\left(\left\|\sum_{i \leq n} a_{i} x_{i}\right\| ; n \in w\right)$ is bounded there exists an $x \in B$ such that $x=\sum_{i \in w} a_{i} x_{i}$; it will be called shrinking if $\lim _{n \in w}\left\|x^{*}\right\|_{n}=0$ for each $x^{*} \in B^{*}$ where $\left\|x^{*}\right\|_{n}=\sup \left\{x^{*}(x): x=\sum_{i>n} a_{i} x_{i}\right.$ and $\left.\|x\| \leqq 1\right\}$, and monotone if for each $x=\sum_{i \in w} a_{i} x_{i}$ in $B\left\|\sum_{i \unlhd m} a_{i} x_{i}\right\|$ is a nondecreasing function of $m$.

A Banach space is smooth if for each $x \in B$ with $\|x\|=1$ there exists a unique $x^{*} \in B^{*}$ such that $\left\|x^{*}\right\|=1$ and $x^{*}(x)=1$. If for each $x^{*} \in B^{*}$ with $\left\|x^{*}\right\|=1$ there exists at most one $x \in B$ such that $\|x\|=1$ and $x^{*}(x)=1$, then the space is called rotund. It is easy to see that this terminology is equivalent to that of [2]. Finally a Banach space is

Received by the editors July 22, 1960 and, in revised form, November 21, 1960. 
called subreflexive [6] if the set of bounded linear functionals which attain their supremum on the unit sphere of $B$ is dense in $B^{*}$.

3. Basis in quasi-reflexive spaces. If $B$ is a quasi-reflexive space of order $n$ then, by definition, we write $B^{* *}=\pi B \oplus F$ where $F$ is a finite $n$-dimensional subspace. It follows that if $\left(x_{i} ; i \in w\right)$ is a basis for $B$ then the sequence $\left(y_{1}^{* *}, \cdots, y_{n}{ }^{* *}, \pi x_{i} ; i \in w\right)$, where $y_{1}^{* *}, \cdots, y_{n}^{* *}$ is a basis for $F$, is a basis for $B^{* *}$ which we will call a basis corresponding to $\left(x_{i} ; i \in w\right)$.

3.1. Theorem. Let $B$ be a quasi-reflexive space. If $B$ has a basis, then this basis is shrinking (boundedly complete) if and only if the corresponding basis in $B^{* *}$ is shrinking (boundedly complete).

Proof. If $\left(x_{i} ; i \in w\right)$ is a shrinking basis for $B$ and $\left(y_{1}^{* *}, \cdots, y_{n}^{* *}\right.$, $\left.\pi x_{i} ; i \in w\right)$ a corresponding basis for $B^{* *}$, then for $x^{* * *} \in B^{* * *}$ we have

$$
\lim _{m}\left\|x^{* * *}\right\|_{m}=\lim _{m ; m>n}\left\|x^{* * *}\right\|_{m} \leqq \lim _{m} \sup _{\|x\| \leqq 1}\left\{x^{* * *}(\pi x): x=\sum_{i>m} a_{i} x_{i}\right\} .
$$

By Theorem $15[3], B^{* * *}=\pi_{1} B^{*} \oplus(\pi B)^{+}$, where $\pi_{1}$ is the canonical isomorphism of $B^{*}$ into $B^{* * *}$, so if $x^{* * *}=\pi_{1} x^{*}+y^{* * *}$, where $x^{*} \in B^{*}$ and $y^{* * *} \in(\pi B)^{+}$we obtain

$$
\lim _{m}\left\|x^{* * *}\right\|_{m} \leqq \lim _{m} \sup _{\|x\| \leqq 1}\left\{x^{*}(x): x=\sum_{i>m} a_{i} x_{i}\right\}=\lim _{m}\left\|x^{*}\right\|_{m}=0 .
$$

The corresponding basis for $B^{* *}$ is therefore shrinking.

If $\left(x_{i} ; i \in w\right)$ is a boundedly complete basis for $B$, let $\left(a_{i} ; i \in w\right)$ be a sequence of real numbers such that the sequence

$$
\left(\left\|\sum_{i \leq \min (m, n)} a_{i} y_{i}^{* *}+\sum_{n<i \leq m} a_{i} \pi x_{i-n}\right\| ; m \in w\right)
$$

is bounded. Since $B$ is quasi-reflexive there exists a continuous projection of $B^{* *}$ onto $\pi B$, the sequence $\left\{\left\|\sum_{n<i \leq m} a_{i} \pi x_{i-n}\right\| ; m \in w\right\}$ $=\left\{\left\|\sum_{i \leq m} a_{n+i} x_{i}\right\| ; m \in w\right\}$ is bounded and there exists $x \in B$ such that $x=\sum_{i \in w} a_{n+i} x_{i}$. Let $y^{* *}=\sum_{i \leqq n} a_{i} y_{i}^{* *}+\pi x$, then $y^{* *}$ $=\sum_{i \leq n} a_{i} y_{i}^{* *}+\sum_{n<i} a_{i} \pi x_{i-n}$ and the corresponding basis in $B^{* *}$ is therefore boundedly complete. The converse in each case is clear.

3.2. Corollary. Let $B$ be a quasi-reflexive space with a monotone shrinking basis; then $B^{(n)}$ is subreflexive for all $n \geqq 0$ where $B^{(0)}=B$ and $B^{(n)}=\left(B^{(n-1)}\right)^{*}$.

Proof. This is a direct consequence of [6] and the above theorem. 
3.3. TheOREM. Let $B$ be a quasi-reflexive space. If $B$ has a boundedly complete basis then $B^{*}$ has a shrinking basis.

Proof. Let $\left(x_{i} ; i \in w\right)$ be a boundedly complete basis for $B$. Let $\left(x_{i}^{*} ; i \in w\right)$ be the sequence in $B^{*}$ biorthogonal to $\left(x_{i} ; i \in w\right)$ and $A$ the closed subspace of $B^{*}$ spanned by $\left(x_{i}^{*} ; i \in w\right)$. Then by Lemma 2 $\left[2\right.$, p. 70], $B^{* *}=\pi B \oplus A^{+}$and since $B$ is quasi-reflexive $A^{+}$must be finite $n$-dimensional; let $y_{1}^{* *}, \cdots, y_{n}{ }^{* *}$ be a basis for $A^{+}$and pick $y_{1}^{*}, \cdots, y_{n}^{*}$ in $B^{*}$ such that $y_{i}^{* *}\left(y_{j}^{*}\right)=\delta_{i j}$. Let $R$ be the subspace spanned by $y_{1}^{*}, \cdots, y_{n}{ }^{*}$, then $B^{*}=R \oplus A$ and $\left(y_{1}^{*}, \cdots, y_{n}{ }^{*}, x_{i}^{*} ; i \in w\right)$ is a basis for $B^{*}$, since $\left(x_{i}^{*} ; i \in w\right)$ is a basis for $A$.

Let $\left(x_{i}^{* *} ; i \in w\right)$ be the sequence in $B^{* *}$ biorthogonal to $\left(y_{1}^{*}, \cdots, y_{n}^{*}, x_{i}^{*}, i \in w\right)$. We will show that $\left(x_{i}^{* *} ; i \in w\right)$ spans $B^{* *}$; by Lemma $1\left[2\right.$, p. 70] this will prove that the basis for $B^{*}$ is shrinking. Let $\left(y_{1}^{* *}, \cdots, y_{n}^{* *}, \pi x_{i} ; i \in w\right)$ be the corresponding basis for $B^{* *}$, then one verifies easily that $x_{i}^{* *}=y_{i}^{* *}, i \leqq n$ and $x_{n+i}^{* *}=\pi x_{i}$ $-\sum_{k \leqq n} y_{k}^{*}\left(x_{i}\right) x_{k}^{* *}, i \in w$. If $x^{* *} \in B^{* *}, x^{* *}=\pi x+y^{* *}$ with $x \in B$ and $y^{* *}=\sum_{i \leqq n} \beta_{i} x_{i}^{* *} \in A^{+}$and one can write $x^{* *}=\sum_{i \in w} x_{i}^{*}(x) \pi x_{i}$ $+\sum_{i \leqq n} \beta_{i} x_{i}^{* *}=\sum_{i \in w} x_{i}^{*}(x) x_{n+i}^{* *}+\sum_{i \leqq n}\left(\beta_{i}+y_{i}^{*}(x)\right) x_{i}^{* *}$. Therefore the sequence $\left(x_{i}^{* *} ; i \in w\right)$ spans $B^{* *}$ and the basis for $B^{*}$ is shrinking.

3.4. Corollary. Let $B$ be a quasi-reflexive space. If $B$ has a basis which is shrinking (boundedly complete) then there exist quasi-reflexive spaces $B^{(j)}, j \in w$ such that $B^{0}=B, B^{(j+1)}=\left(B^{(j)}\right)^{*}, B^{(2 k)}$ has a shrinking (boundedly complete) basis and $B^{(2 k+1)}$ has a boundedly complete (shrinking) basis.

Proof. The proof follows from Lemma 3.4 [1], Corollary 1 [2, p. 71] and Theorems 3.1 and 3.3 above.

Let $B^{*}$ have a basis. Whether or not this implies the existence of a basis for $B$ appears to be an open question. In case $B$ is quasi-reflexive the following theorem provides a partial answer.

3.5. Theorem. Let $B$ be a quasi-reflexive Banach space. If $B^{*}$ has a boundedly complete basis then $B$ has a shrinking basis.

Proof. Let $\left(x_{i}^{*} ; i \in w\right)$ be a boundedly complete basis. By Lemma $3.4[1] B^{*}$ is quasi-reflexive and so, as in the proof of the preceding theorem $B^{* * *}=\pi_{1} B^{*} \oplus A^{+}$where $A$ is the subspace spanned by $\left(x_{i}^{* *} ; i \in w\right)$ the sequence biorthogonal to $\left(x_{i}^{*} ; i \in w\right)$ and $A^{+}$is finite $n$-dimensional. Also $B^{* *}=R \oplus A$ where $R$ is finite $n$-dimensional and $A$ is a total subspace of $B^{* *}$. By Theorems 14 and 16 of [3] there exists an isomorphism $T$ from $B^{*}$ onto $A^{*}$ such that $\left(T x^{*}\right)\left(y^{* *}\right)=y^{* *}\left(x^{*}\right)$ for all $x^{*} \in B^{*}$ and $y^{* *} \in A$. By Theorem 3.6 of [1] there exists an 
isomorphism $S$ of $A$ onto $B$. It is easy to verify that the sequence $\left(S x_{i}^{* *} ; i \in w\right)$ is a basis for $B$. To see that this basis is shrinking consider the sequence $\left(z_{i}^{*} ; i \in w\right)$ in $B^{*}$ biorthogonal to $\left(S x_{i}^{* *} ; i \in w\right)$. The sequence $\left(T x_{i}^{*} ; i \in w\right)$ in $A^{*}$ is biorthogonal to $\left(x_{i}^{* *} ; i \in w\right)$, therefore $S y^{* *}=S \sum_{i \in w}\left(T x_{i}^{*}\right)\left(y^{* *}\right) x_{i}^{* *}=\sum_{i \in w} y^{* *}\left(x_{i}^{*}\right) S x_{i}^{* *}$ for all $y^{* *} \in A$; it follows that $z_{i}^{*}\left(S y^{* *}\right)=y^{* *}\left(x_{i}^{*}\right)$ for all $i \in w$ and all $y^{* *} \in A$; since $A$ is total we have $T^{-1} S^{*} z_{2}^{*}=x_{i}^{*}$ and so $\left(z_{i}^{*} ; i \in w\right)$ is the isomorphic image of the basis $\left(x_{i}^{*} ; i \in w\right)$. We conclude that $\left(S x_{i}^{* *} ; i \in w\right)$ is a shrinking basis for $B$.

If $B$ is a reflexive space and $B$ has a basis, then it is well known (see, for example, [2]) that this basis must be both shrinking and boundedly complete. The following theorem holds for quasi-reflexive spaces.

3.6. Theorem. Let $B$ be a quasi-reflexive space of order 1, then any basis for $B$ is either shrinking or boundedly complete but not both.

Proof. Let $\left(x_{i} ; i \in w\right)$ be a basis for the space $B$ which is quasireflexive of order 1 . If $\left(x_{i} ; i \in w\right)$ is not shrinking, then, by Lemma 1 $\left[2\right.$, p. 70], $A$ the closed subspace spanned by $\left(x_{i}^{*} ; i \in w\right)$, the sequence in $B^{*}$ biorthogonal to $\left(x_{i} ; i \in w\right)$, is a proper subspace of $B^{*}$ and consequently $A^{+}$is nonvoid. Since $B$ has order 1 we can write $B^{* *}$ $=\pi B \oplus\left\{a y^{* *}\right\}, y^{* *} \bigoplus \pi B, a$ any real number. Let $z_{0}^{* *} \in A^{+}$; then $z_{0}^{* *}=\pi x_{0}+a_{0} y^{* *}$ for some $x_{0} \in B$ and $a_{0} \neq 0$, and for any $x^{*} \in A$ we have $x^{*}\left(x_{0}\right)=-a_{0} y^{* *}\left(x^{*}\right)$. If $z^{* *}=\pi x+a y^{* *} \in A^{+}$we must have $x^{*}(x)=\left(a / a_{0}\right) x^{*}\left(x_{0}\right)$ for all $x^{*} \in A ; A$ being total this implies that $x=\left(a / a_{0}\right) x_{0}$ and so $z^{* *}=\left(a / a_{0}\right)\left(\pi x_{0}+a_{0} y^{* *}\right)=\left(a / a_{0}\right) z_{0}^{* *}$. We conclude that $A^{+}=\left\{a z_{0}^{* *}\right\}$ is 1-dimensional. It is easy to see that one can write $B^{* *}=\pi B \oplus A^{+}$. By Lemma $2\left[2\right.$, p. 70] this implies that $\left(x_{i} ; i \in w\right)$ is boundedly complete. Finally if $\left(x_{i} ; i \in w\right)$ were both shrinking and boundedly complete then $B$ would be reflexive by Theorem 3 [2, p. 71], contradicting the hypothesis.

4. The supremum of functionals in quasi-reflexive spaces. James [4] has shown that if all bounded linear functionals on a separable Banach space attain their supremum on the unit sphere then the space is reflexive. Whether this result is true for a general Banach space is not known. However the result can be extended to nonseparable quasi-reflexive Banach space.

4.1. Theorem. Let $B$ be a quasi-reflexive Banach space. If all bounded linear functionals on $B$ attain their supremum on the unit sphere of $B$ then $B$ is reflexive.

Proof. Assume the hypothesis of the theorem. If $B$ is separable then 
James' result can be applied. If $B$ is not separable then by Theorem 4.6 of [1] there exists a nonseparable reflexive subspace $Z$ such that $B / Z$ is separable.

The mapping $T$ of $Z^{+}$onto $(B / Z)^{*}$ defined by $T x^{*}(x+Z)=x^{*}(x)$ for all $x^{*} \in Z^{+}$and $x \in B$, is an isometric isomorphism. If $x^{*} \in Z^{+}$ there exists $x_{0} \in B$ such that $\left\|x_{0}\right\|=1$ and $x^{*}\left(x_{0}\right)=\left\|x^{*}\right\|$, and therefore $T x^{*}\left(x_{0}+Z\right)=x^{*}\left(x_{0}\right)=\left\|x^{*}\right\|=\left\|T x^{*}\right\|$. Every functional on $B / Z$ attains its supremum on the unit sphere of $B / Z$ consequently $B / Z$, as well as $Z$ is reflexive. It follows that $B$ is reflexive.

\section{Rotundity and smoothness in quasi-reflexive spaces.}

5.1. Theorem. If $B$ is a quasi-reflexive Banach space, there exists an equivalent norm for $B$ such that if $B$ is either rotund or smooth in this norm, then $B$ is reflexive.

Proof. By Theorem 3.5 [1] there exists an equivalent norm for $B$ and quasi-reflexive Banach spaces $B^{(-6)}, B^{(-4)}$ and $B^{(-1)}$ such that $\left(B^{(-5)}\right)^{* * * *}=B^{(-1)},\left(B^{(-4)}\right)^{* * * *}=B$ and $\left(B^{(-1)}\right) *=B$. By Theorem 20 [3], if $B$ is rotund in this norm we must have $B^{(-4)}$ reflexive. It follows that $B$ is reflexive. If $B$ is smooth, then by $\left[5\right.$, p. 12] $B^{(-1)}$ is rotund and so $B^{(-5)}$ is reflexive; $B$ is therefore reflexive.

\section{BiBLIOGRAPHY}

1. Paul Civin and Bertram Yood, Quasi reflexive spaces, Proc. Amer. Math. Soc. vol. 8 (1957) pp. 906-911.

2. Mahlon M. Day, Normed linear spaces, Berlin, Springer-Verlag, 1958.

3. J. Dixmier, Sur un théorème de Banach, Duke Math. J. vol. 15 (1948) pp. 10571071.

4. Robert C. James, Reflexivity and the supremum of linear functionals, Ann. of Math. vol. 66 (1957) p. 159.

5. V. L. Klee, Convex bodies and periodic homeomorphisms in Hilbert space, Trans. Amer. Math. Soc. vol. 74 (1953) pp. 10-43.

6. R. R. Phelps, Subreflexive normed linear spaces, Arch. Math. vol. 8 (1957) pp. 444-450.

Canadian Mathematical Congress, Kingston and UNIVERSITY OF SASKATCHEWAN 